\title{
Strangeness Production in Microscopic Transport Models
}

\author{
Steffen A. Bass $\dagger$ \\ Department of Physics, Duke University \\ \& RIKEN-BNL Research Center, Brookhaven National Laboratory
}

\begin{abstract}
Strangeness production in microscopic transport models for relativistic heavy-ion collisions from SIS to RHIC is reviewed: after a brief introduction into elementary strangeness production processes, the main emphasis is put on strangeness as indicator of the nuclear equation of state, the excitation function of the $K^{+} / \pi^{+}$ ratio and strangeness as a deconfinement indicator.
\end{abstract}

\section{Introduction and overview}

Microscopic transport models are a unique tool for the study of relativistic heavy-ion collisions: they offer a method of connecting the observable final state of such a collision with the time-evolution of the reaction, its dynamics and many not directly observable but very much sought after quantities and phenomena like the nuclear equation of state and the deconfinement phase-transition to a Quark-Gluon-Plasma [1]. Progress in heavy-ion physics requires a symbiosis between experiment and (transport-)theory.

The basic principles of microscopic transport models can best be explained by studying the Vlasov-Uehling-Uhlenbeck (VUU) equation:

$$
\begin{aligned}
{\left[\frac{\partial}{\partial t}+\right.} & \left.\left(\frac{\mathbf{p}_{1}}{m}+\vec{\nabla}_{p_{1}} \Re \Sigma^{+}\right) \cdot \vec{\nabla}_{r_{1}}-\vec{\nabla}_{r_{1}} \Re \Sigma^{+} \cdot \vec{\nabla}_{p_{1}}\right] f_{1}\left(\mathbf{r}_{1}, \mathbf{p}_{1}, t\right) \\
= & \frac{2 g}{m^{2}(2 \pi \hbar)^{3}} \int \mathrm{d}^{3} \mathbf{p}_{2} \int \mathrm{d}^{3} \mathbf{p}_{1}^{\prime} \int \mathrm{d}^{3} \mathbf{p}_{2}^{\prime} \delta^{4}\left(p_{1}+p_{2}-p_{1}^{\prime}-p_{2}^{\prime}\right) \frac{\mathrm{d} \sigma}{\mathrm{d} \Omega} \\
& \times\left[f_{1}^{\prime} f_{2}^{\prime}\left(1-f_{1}\right)\left(1-f_{2}\right)-f_{1} f_{2}\left(1-f_{1}^{\prime}\right)\left(1-f_{2}^{\prime}\right)\right]
\end{aligned}
$$

This equation describes the time-evolution of the one-particle distribution function $f_{1}\left(\mathbf{r}_{1}, \mathbf{p}_{1}, t\right)$ which contains the positions and momenta of all particles of the system (i.e. the microscopic degrees of freedom) - initially all the protons and neutrons (or, in the case of a partonic description, the quarks and gluons) of the two colliding nuclei. These particles may interact either through an interaction given by the the real part of the retarded self-energy $\Re \Sigma^{+}$(often also approximated by and referred to as the mean field) or through binary scattering, symbolized in this equation by the differential cross † bass@phy.duke.edu 


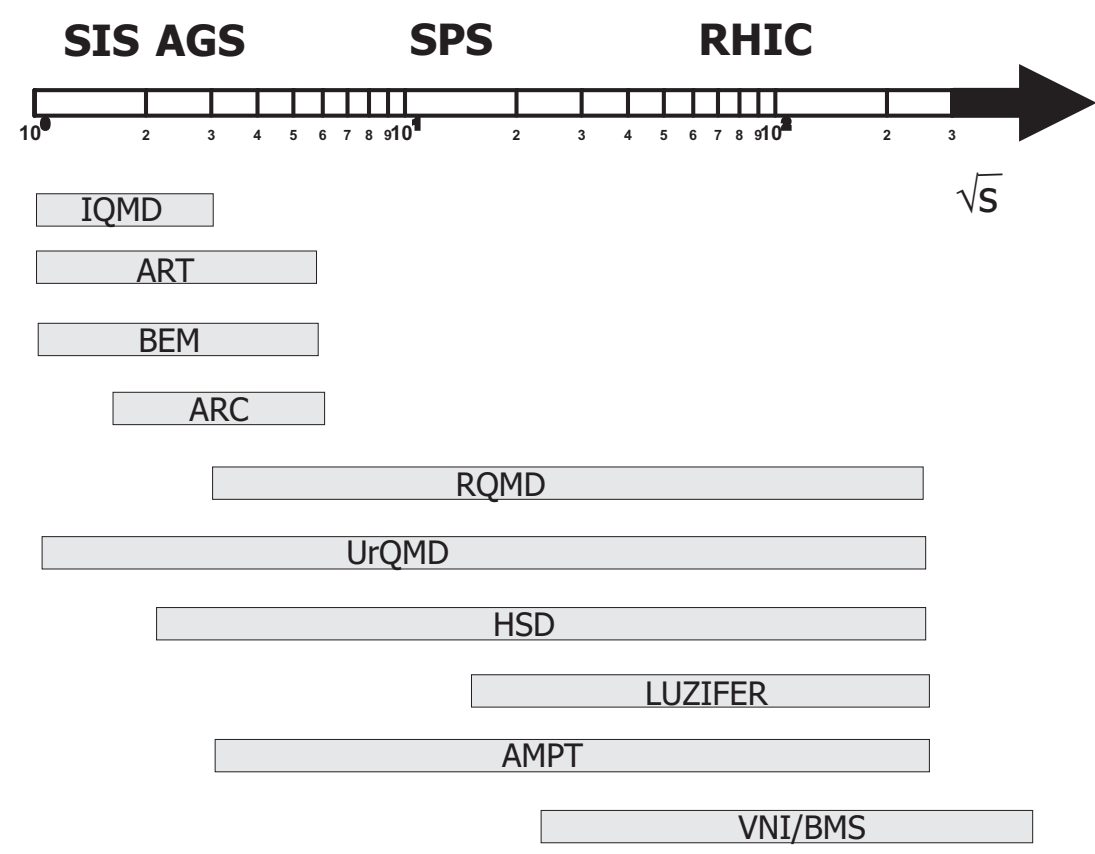

Figure 1. Overview of available microscopic transport models and their range of applicability in incident beam energy.

section $\frac{\mathrm{d} \sigma}{\mathrm{d} \Omega}$. If the r.h.s of this equation, the collision integral, is neglected one obtains the Vlasov equation. On the other hand, if the real part of the retarded self-energy $\Re \Sigma^{+}$ is neglected and if the so called Pauli blocking factors $(1-f)$ in the collision integral are approximated by 1, one obtains the famous Boltzmann equation.

In terms of the details of the implementation of mean field and collision term (e.g. in the number of hadronic resonances included or in the parametrization of cross sections), transport models may vary widely. Figure 1 provides an overview of currently available microscopic transport models applicable from SIS up to RHIC energies: models with purely hadronic degrees of freedom utilizing both, a mean field as well as a collision term are (I)QMD [2], VUU/BUU [3], ART [4] and BEM [5]. Models not containing a mean field are commonly referred to as cascade models - ARC [6] and LUZIFER [10] fall into this category. At higher energies initial particle production requires the introduction of string excitations - models which combine strings and hadrons are RQMD [7], UrQMD [8] and HSD [9]. At RHIC energies strings and hadrons may not anymore be the relevant degrees of freedom - here deconfinement needs to be taken into account and the elementary degrees of freedom for the initial reaction stage are quarks and gluons interacting through hard scattering as in VNI/BMS [11] and AMPT [12].

\section{Mechanisms of strangeness production}

Strangeness may be produced either in initial collisions among the incoming nucleons of the two colliding nuclei or through secondary interactions among produced particles, 

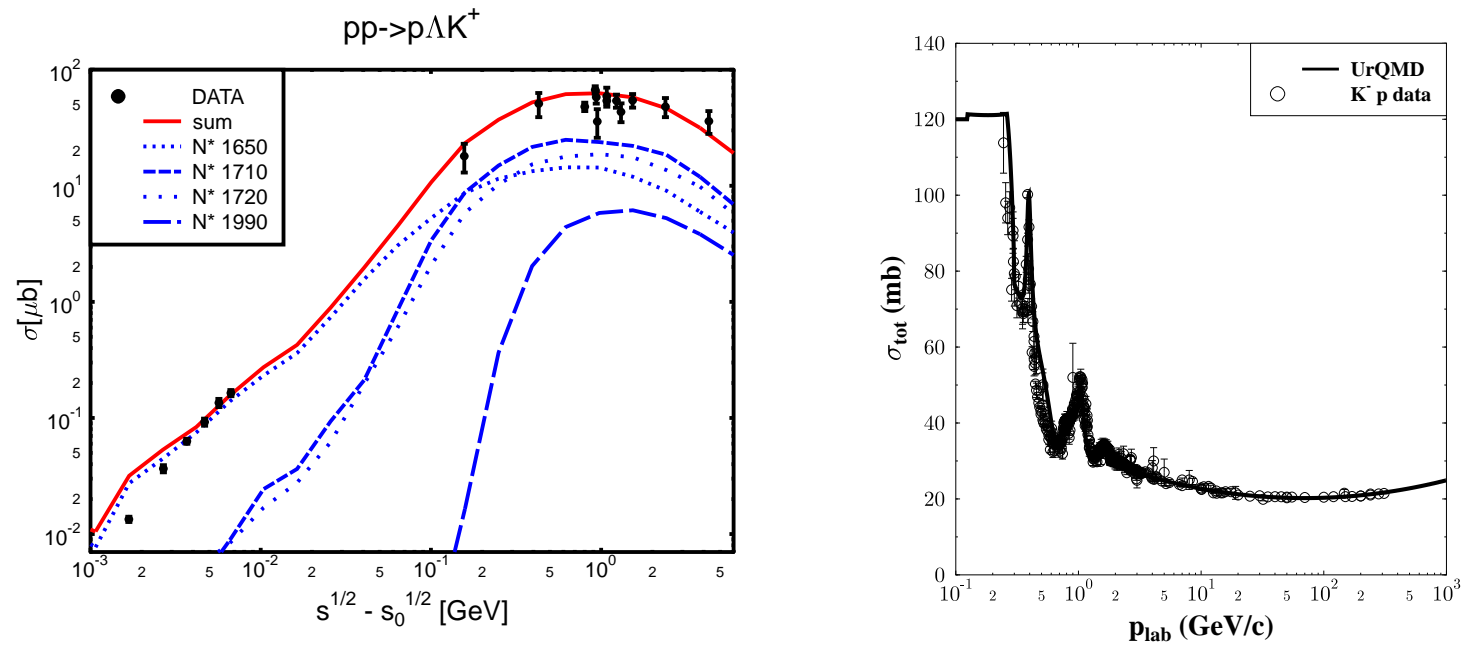

Figure 2. Left: excitation function of the exclusive $p+p \rightarrow p+\Lambda+K^{+}$production cross section close to threshold in a resonance model. Right: $K^{-}+p$ scattering cross section in UrQMD compared to data.

e.g. pions and nucleons or excited resonances.

\subsection{Strangeness production at threshold}

There are two different approaches for initial strangeness production close to threshold:

(i) direct parametrization: the individual cross sections for different exclusive production channels, e.g. $p+p \rightarrow p+\Lambda+K^{+}$are directly parameterized. This method is very accurate if the cross sections are well known (i.e. well measured) but may become cumbersome away from threshold with increasing number of explicit channels to parameterize.

(ii) resonance model: here strangeness production is a two step process. Initially a heavy baryon resonance is excited, e.g. via $p+p \rightarrow N+N_{1710}^{*}$, which subsequently decays via $N_{1710}^{*} \rightarrow Y+K^{+}$. This approach allows for an easier extension into the higher energy domain and may provide some rudimentary guidance for unknown strangeness production cross sections in secondary collisions, e.g. $\pi+N \rightarrow N_{1710}^{*} \rightarrow$ $Y+K^{+}$. The left frame of figure 2 shows a fit of the resonance model to the exclusive $p+p \rightarrow p+\Lambda+K^{+}$reaction channel.

Secondary interactions like pion-induced strangeness production or flavor-exchange reactions are at least as important for the reaction dynamics and final strangeness yield as the initial/primordial strangeness production channels. The right frame of figure 2 shows the $K^{-}+N$ reaction cross section, which exhibits a distinct resonance structure. The hyperon resonances which are excited via this cross section may then either decay again into the $K^{-}+N$ channel, or to almost equal probability decay into the $Y+\pi$ channel, thus transferring strangeness in and out of baryonic degrees of freedom. As we shall later see, this $K^{-}+N \leftrightarrow Y+\pi$ exchange reaction is of particular importance. 

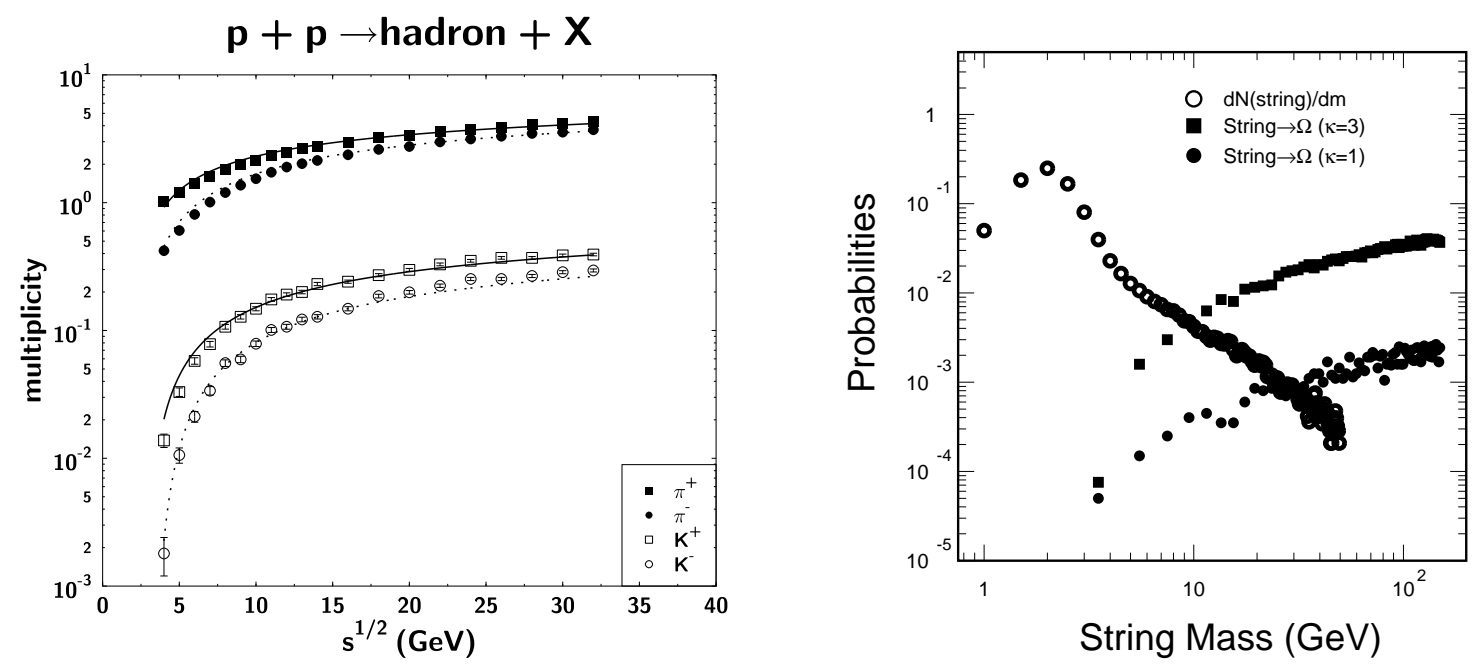

Figure 3. Left: excitation function of the $K$ and $\pi$ multiplicity in elementary $p+p$ reactions in UrQMD at large CM-energies compared to data. Right: distribution of string masses in $\mathrm{Pb}+\mathrm{Pb}$ reactions at the CERN-SPS $(160 \mathrm{GeV} / \mathrm{u})$ and the $\Omega^{-}$ production probability as a function of string-mass for two different values of the string-tension $\kappa$.

Ambiguities in both types of approaches arise from unknown and mostly immeasurable cross sections such as strangeness production in interactions involving baryon- and meson-resonances, e.g. $\Delta_{1232}+N \rightarrow K^{+}+X$.

\subsection{Strangeness production at high energies}

At higher incident beam energies, particle production in general is dominated by string excitation and fragmentation. Up to $70 \%$ of the total strangeness produced in a $\mathrm{Pb}+\mathrm{Pb}$ collision at top CERN-SPS energies is produced in initial highly energetic nucleonnucleon interactions which lead to the excitation and subsequent fragmentation of strings [8]. On an elementary hadron-hadron level, the parameters of the string fragmentation are fitted to measured multiplicities and momentum distributions. The left frame of figure 3 shows the resulting excitation function of the pion and kaon multiplicity in proton-proton reactions in the UrQMD model (plot symbols are data, lines the model fit).

When going from elementary hadron-hadron interactions to collisions of heavy nuclei one can explore the possibility of medium effects due to the hot and dense environment of the reaction. One such (non-hadronic) medium effect is the formation of color-ropes [13] - overlapping strings form a chromo-electric field which due to its larger field strength compared to the individual color-fluxtubes has an enhanced probability of fragmenting into strange hadrons. Rope effects can be simulated by increasing the string-tension $\kappa$ from it's vacuum value of $\kappa=1 \mathrm{GeV} / \mathrm{fm}$ to a value of $\kappa=3 \mathrm{GeV} / \mathrm{fm}$ for strings fragmenting in the high energy-density region of the collision. The right frame of figure 3 shows the distribution of string masses excited in a $\mathrm{Pb}+\mathrm{Pb}$ collision 

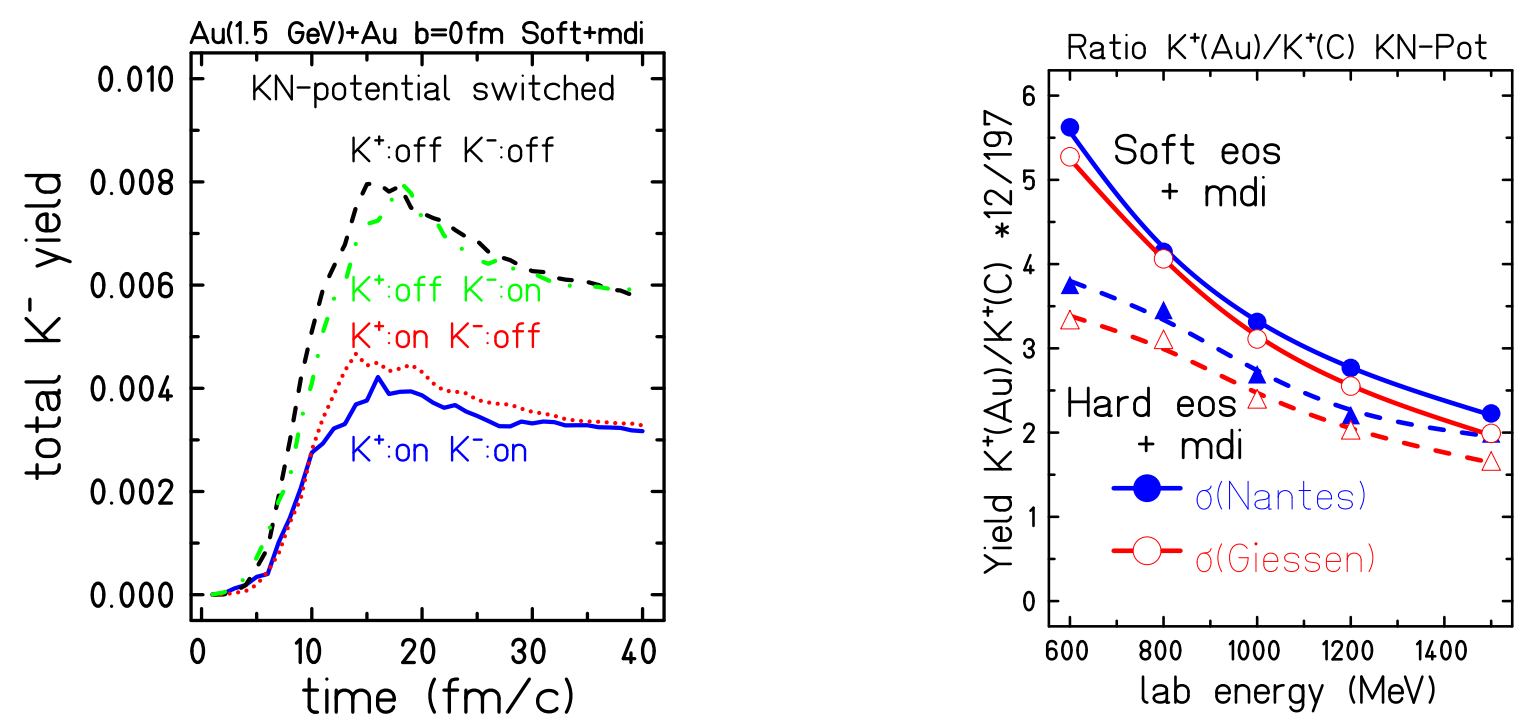

Figure 4. Left: time-evolution of the $K^{-}$yield with and without inclusion of $K^{ \pm}$ potentials. Right: ratio of energy-spectra of $K^{+}$in $\mathrm{Au}+\mathrm{Au}$ and $\mathrm{C}+\mathrm{C}$ collisions for different cross section and equation of state parametrizations.

at $160 \mathrm{GeV} / \mathrm{u}$ as well as the fragmentation probability of the string into an $\Omega^{-}$, both for $\kappa=1 \mathrm{GeV} / \mathrm{fm}$ as well as for $\kappa=3 \mathrm{GeV} / \mathrm{fm}$ [14]. A drastic enhancement of the $\Omega^{-}$ formation probability by more than one order of magnitude is visible.

\section{Strangeness and the nuclear equation of state}

In the SIS and AGS energy domain the main issues to be addressed by microscopic transport theory with respect to strangeness are the existence of in-medium modifications for kaons and the nuclear equation of state. In particular the $K^{-}$is considered to be strongly influenced by a scalar as well as a vector interaction. The left frame of figure 4 shows the time-evolution of the $K^{-}$yield for different combinations of potentials acting upon the $K^{-}$or the $K^{+}$[15]. Surprisingly the $K^{-}$yield is totally insensitive to $K^{-}$potentials but very sensitive to $K^{+}$potentials. This flavor coupling can be well understood through the flavor-exchange reaction $K^{-}+N \leftrightarrow Y+\pi$ with the the hyperons being linked to the $K^{+}$and their potential via associated production or higher resonance states like $N_{1710}^{*} \leftrightarrow Y+K^{+}$. The main observable to explore potential effects on $K^{-}$is the so called kaon-flow, which has been shown to be extremely sensitive to the strength and nature of the real part of the kaon-nucleon interaction [16].

The $K^{+}$yield, on the other hand, has been found to be very sensitive to the nuclear equation of state [17]. Ambiguities may arise due to different implementations of unknown production cross sections like $\Delta_{1232}+N \rightarrow N+Y+K^{+}$. In order to avoid these, ratios of $K^{+}$spectra for heavy and light collision systems can be used [18]. The right frame of figure 4 shows the ratio of the $K^{+}$energy spectra for $\mathrm{Au}+\mathrm{Au}$ and $\mathrm{C}+\mathrm{C}$ collisions for different cross section and equation of state parametrizations [19]. A strong sensitivity to the equation of state is observed while different production 
Strangeness Production in Microscop
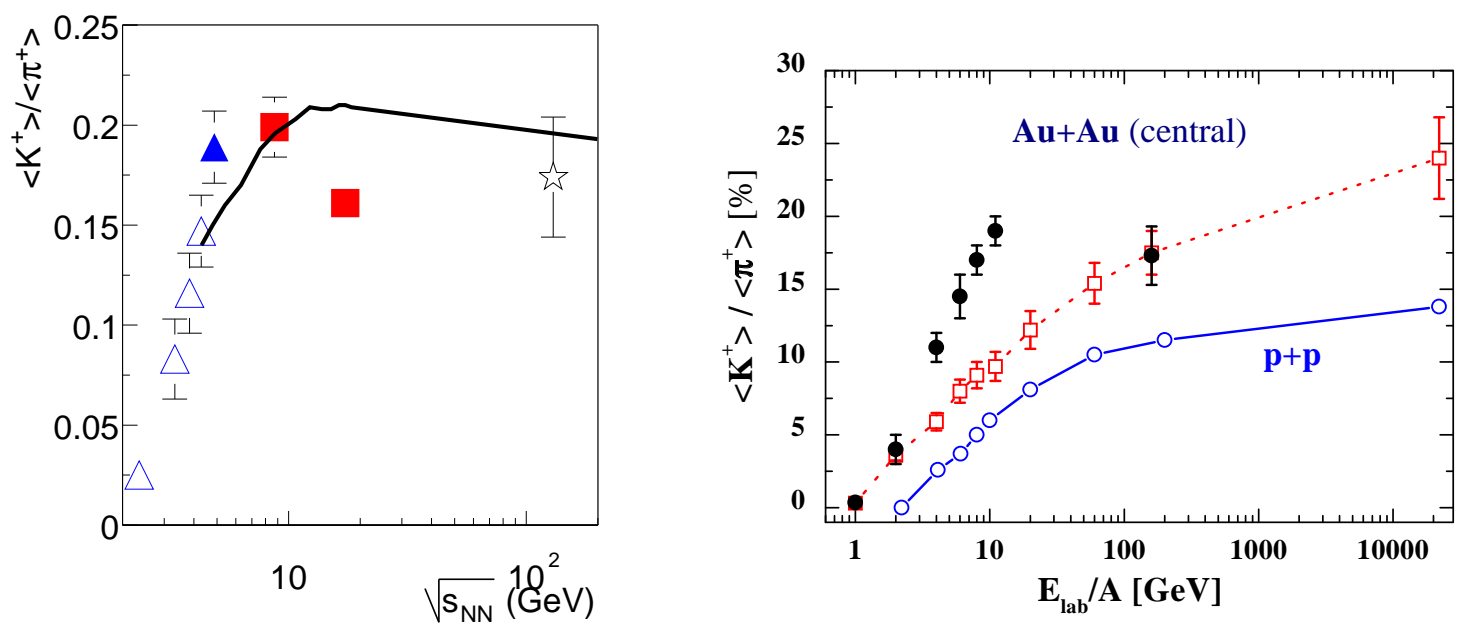

Figure 5. Excitation function of the $K^{+} / \pi^{+}$ratio in RQMD (left) and HSD (right; open symbols refer to HSD, closed symbols are data).

cross sections do not alter the value or functional form of the ratio. Comparisons of current calculations with data favor a soft equation of state with momentum-dependent interaction [18].

\section{Excitation functions}

The $K^{+} / \pi^{+}$ratio provides a measure of the ratio of newly produced strange to nonstrange valence-quarks in a heavy-ion reaction. A kink in its excitation function vs. incident beam energy was thought to hint at a possible deconfinement phase-transition. Recent calculations in the framework of a statistical model show that the $K^{+} / \pi^{+}$ratio is expected to reach its maximum value around a beam energy of $30 \mathrm{GeV} / \mathrm{u}$, well in line with experimental findings [20]. While the agreement of the data with statistical model calculations cannot per se prove or disprove the existence of a deconfinement phase-transition, the $K^{+} / \pi^{+}$ratio as deconfinement indicator remains ambiguous at best.

However, the beam-energy dependence of the $K^{+} / \pi^{+}$ratio has emerged as a rather stringent test for microscopic transport models: figures 5 and 6 show the excitation function of the $K^{+} / \pi^{+}$ratio for RQMD (fig. 5 left, provided by [22]), HSD (fig 5 right, taken from [23]) and UrQMD (fig. 6 left, provided by [21, 22]). While RQMD compares well to the data, both HSD and UrQMD exhibit problems which may not necessarily be rooted explicitly in the strangeness production mechanisms of the particular models: in the case of HSD, an analysis shows that the observed functional dependence of the $K^{+} / \pi^{+}$ratio is very sensitive to the threshold of the string production cross section and the excitation of the high-mass resonance continuum [24]. Older versions of RQMD with a similar implementation of these cross sections exhibit the same behavior as observed in HSD. In the case of UrQMD the under-prediction of the $K^{+} / \pi^{+}$ratio starting at AGS energies can be traced to a pion excess (the kaon yields roughly agree with the data). 

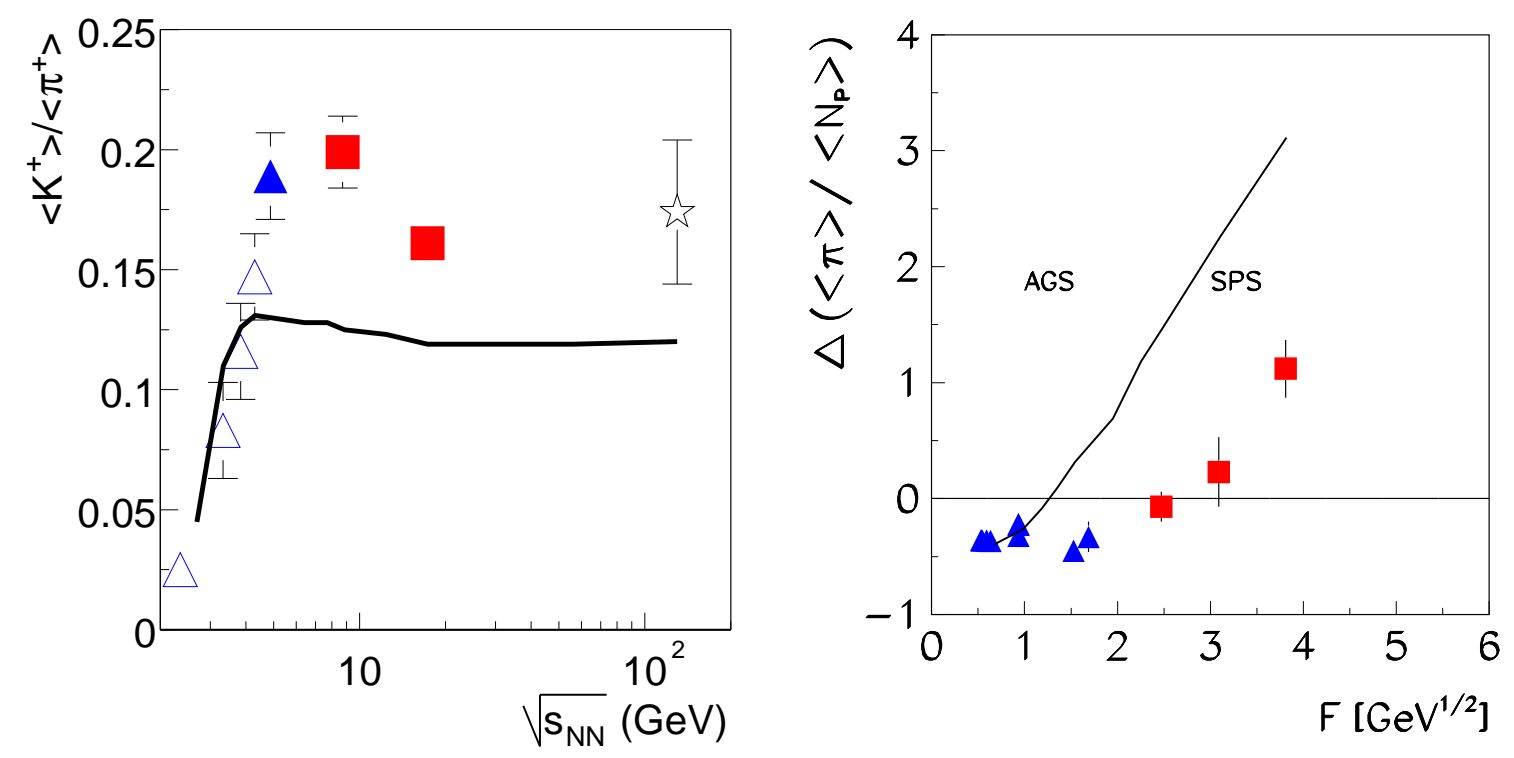

Figure 6. Left: excitation function of the $K^{+} / \pi^{+}$in UrQMD. Right: comparison of the pion per participant excess in $\mathrm{Pb}+\mathrm{Pb}$ reactions vs. $\mathrm{p}+\mathrm{p}$ reactions in UrQMD to data.

The right frame of figure 6 shows the pion per participant excess in $\mathrm{Pb}+\mathrm{Pb}$ reactions vs. $p+p$ reactions in UrQMD compared to data [21, 22]: in UrQMD this excess sets in too early with respect to the incident beam energy. It is very likely that this behavior is caused by the violation of detailed balance due to the lack of multi-particle (mostly pion) collisions which would convert the pion excess into heavier particles such as antibaryons and hyperons $[25,26]$ - preliminary calculations incorporating these processes yield a $K^{+} / \pi^{+}$ratio very close to the data [21].

A more detailed study of various excitation functions of the $K^{+} / \pi^{+}$ratio in RQMD as well as a discussion of the $K^{+} / K^{-}$ratio can be found in [27].

\section{Strangeness as deconfinement indicator}

The relative enhancement of strange and especially multistrange baryons with respect to peripheral (or proton induced) interactions has been suggested as a signature for the transient existence of a QGP-phase [28, 29, 30]: the main argument being that the (chemical or flavor) equilibration times should be much shorter in the plasma phase than in a thermally equilibrated hadronic fireball of $T \sim 160 \mathrm{MeV}$.

The dominant production mechanism in an equilibrated (gluon rich) plasma phase, namely the production of $s \bar{s}$ pairs via gluon fusion $(g g \rightarrow s \bar{s})$ [28], should allow for equilibration times similar to the interaction time of the colliding nuclei, and to the expected plasma lifetime (a few $\mathrm{fm} / \mathrm{c}$ ).

The yields of strange baryons per event calculated in UrQMD are shown in the left frame of figure 7 as a function of the number of participants for $\mathrm{Pb}+\mathrm{Pb}$ and 

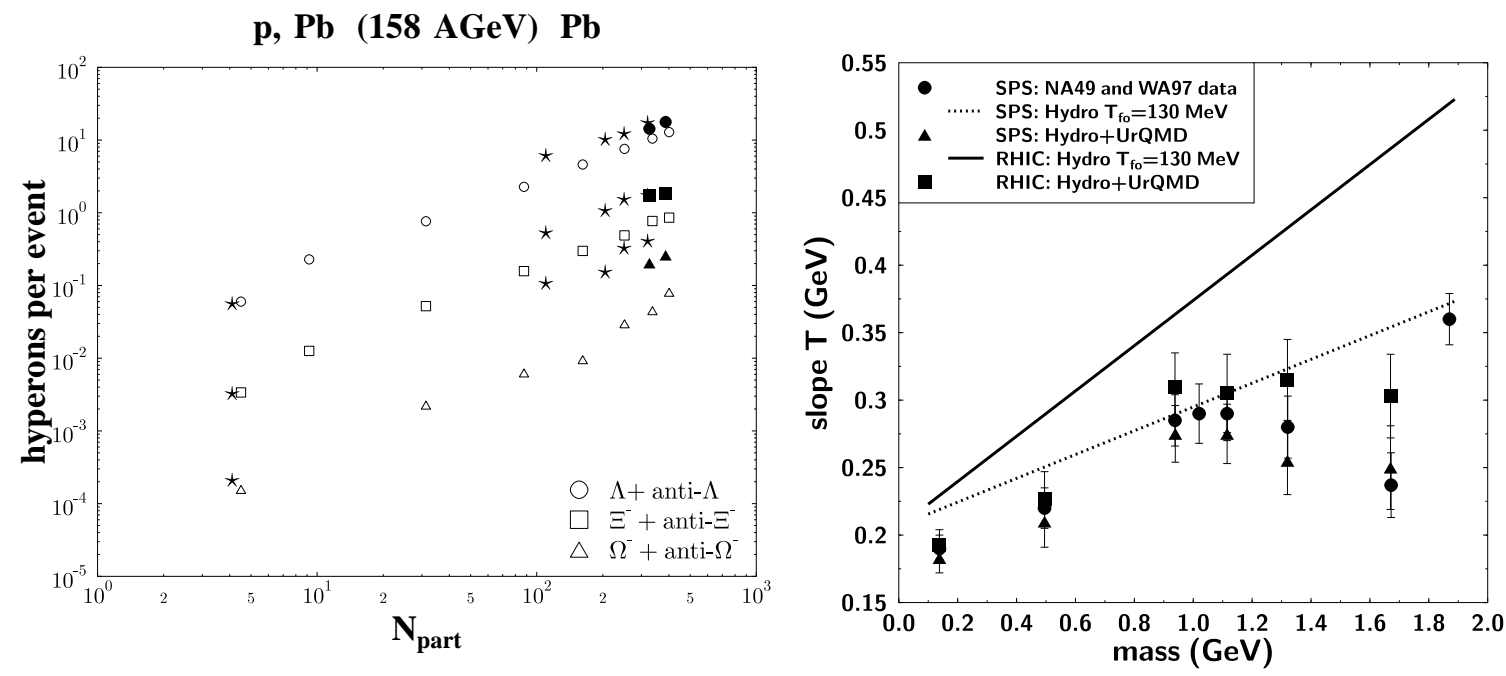

Figure 7. Left: excitation function of strange baryon multiplicity vs. number of participants in UrQMD. Right: Inverse slopes of the $m_{T}$-spectra of $\pi, K, p, \Lambda+\Sigma^{0}$, $\Xi^{0}+\Xi^{-}$, and $\Omega^{-}$at $y_{c . m .}=0$.

$\mathrm{p}+\mathrm{Pb}$ collisions at $160 \mathrm{GeV} / \mathrm{u}[31]$. The $\Lambda+\bar{\Lambda}$ - (circles), $\Xi^{-}+\overline{\Xi^{-}}$- (squares), and $\Omega^{-}+\overline{\Omega^{-}}$- (triangles) values are shown. The stars correspond to experimental data of the WA97 collaboration [32]. Open symbols represent the results of the standard UrQMD calculations, whereas full symbols exhibit a calculation with an enhanced string tension of $\kappa=3 \mathrm{GeV} / \mathrm{fm}$, for the most central collisions $\left(N_{\text {part }} \geq 300\right)$. Obviously the standard UrQMD calculation, which can be seen as a baseline of known hadronic physics, strongly underestimates the (multi-)strange particle yields in central collisions, in particular in the case of the $\Omega^{-}$. Only the inclusion of non-hadronic medium effects, like color-ropes [13], which are simulated by increasing the string-tension for central collisions (see also the right frame of figure 3), enhances the yield to a level of near-compatibility with the data. Similar findings have also been made in the context of HIJING calculations [33]. While these findings by no means prove the validity of the color-rope approach, they clearly show the necessity of some kind medium effect beyond regular binary hadronic (re)scattering in order to understand the data. This statement is corroborated by a calculation with the string model NeXuS, including so-called QGP droplets - domains of high energy-density hadronizing according to a statistical phase-space population which give rise to a similar strangeness enhancement [34].

However, recently hadronic multi-particle interactions in the early dense reaction phase have been suggested to significantly enhance the yield of anti-protons and (anti)hyperons $[25,26]$. It remains to be seen, however, whether these effects are sufficient to explain the observed $\Omega^{-}$enhancement or other non-hadronic (i.e. deconfinement based) effects need to be taken into account.

The study of deconfinement and a subsequent phase-transition to deconfined hadronic matter poses a great challenge to microscopic transport models. In most 
approaches hadronization is a uni-directional process and the equation of state of the system ill-defined. One possible remedy is to use a hydrodynamical approach for the early deconfined phase of the reaction and subsequent phase-transition, coupled with a microscopic calculation for the later, hadronic, reaction phase in which the hydrodynamical assumptions are not valid any longer [35]. In the following, such a combined macro+micro model will be used to study the flavor- and mass-dependence of hadronic slope parameters: the right frame of fig. 7 displays these inverse slope parameters $T$ obtained by an exponential fit to $d N_{i} / d^{2} m_{T} d y$ in the range $m_{T}-m_{i}<$ $1 \mathrm{GeV}$ for SPS and RHIC [36] and compares them to SPS data [32]. The trend of the data, namely the "softer" spectra of $\Xi$ 's and $\Omega$ 's as compared to a linear $T(m)$ relation is reproduced reasonably well. This is in contrast to "pure" hydrodynamics with kinetic freeze-out on a common hypersurface (e.g. the $T=130 \mathrm{MeV}$ isotherm), where the stiffness of the spectra increases linearly with mass as denoted by the lines in fig. 7 . When going from SPS to RHIC energy, such a hybrid model as discussed here generally yields only a slight increase of the inverse slopes, although the specific entropy is larger by a factor of 4-5! The reason for this behavior is the first-order phase transition that softens the transverse expansion considerably.

The reason for the softening of the spectra is that the hadron gas emerging from the hadronization of the QGP is almost "transparent" for the multiple strange baryons. Analyzing the collision numbers, one finds that $\Omega$ 's suffer on average only one hadronic interaction, whereas $N$ 's and $\Lambda$ 's suffer far more collisions with other hadrons before they freeze-out. Thus, one may conclude that the spectra of $\Xi$ 's and especially $\Omega$ 's

are practically unaffected by the hadronic reaction stage and closely resemble those on the phase boundary. They therefore act as probes of the QGP expansion prior to hadronization and can be used to measure the expansion rate of the deconfined phase.

\section{Acknowledgments}

I wish to thank M. Bleicher, R. Bramm, W. Cassing, J. Cleymans, A. Dumitru, C. Fuchs, M. Gazdzicki, C. Greiner, C. Hartnack, C.M. Ko, T. Kolleger, S. Pal, S. Soff, S. Vance, H. Weber and N. Xu for many helpful discussions and their support during the preparation of this review. This work was supported by RIKEN, Brookhaven National Laboratory and DOE grants DE-FG02-96ER40945 as well as DE-AC02-98CH10886.

\section{References}

[1] for reviews on QGP signatures, see

J. Harris and B. Müller, Ann. Rev. Nucl. Part. Sci. 46 (1996) 71.

S.A. Bass, M. Gyulassy, H. Stöcker and W. Greiner, J. Phys. G25 (1999) R1.

[2] C. Hartnack, Ph.D. thesis, Goethe University Frankfurt, 1992;

S. A. Bass, C. Hartnack, H. Stocker and W. Greiner, Phys. Rev. C51, 3343 (1995).

[3] H. Kruse, B.V. Jacak, and H. Stöcker, Phys. Rev. Lett. 54, 289 (1985);

J. Aichelin and G. Bertsch, Phys. Rev. C31, 1730 (1985);

J.J. Molitoris and H. Stöcker, Phys. Rev C32, R346 (1985); 
K. Weber, B. Blattel, V. Koch, A. Lang, W. Cassing, and U. Mosel, Nucl. Phys. A515, 747 (1990).

[4] B.A. Li and C. M. Ko, Phys. Rev. C52, 2037 (1995);

B.A. Li, Int. Journal of Modern Physics E10 (2001) 267.

[5] P. Danielewicz, Nucl. Phys. A673, 375 (2000).

[6] Y. Pang, T. J. Schlagel and S. H. Kahana, Phys. Rev. Lett. 68, 2743 (1992).

[7] H. Sorge, H. Stocker and W. Greiner, Annals Phys. 192, 266 (1989).

[8] S. A. Bass et al., Progr. Part. Nucl. Physics Vol. 41 (1998) 225;

M. Bleicher et al., J. Phys. G25 (1999) 1859

[9] W. Ehehalt and W. Cassing, Nucl. Phys. A602, 449 (1996).

[10] D. E. Kahana and S. H. Kahana, Phys. Rev. C58, 3574 (1998).

[11] K. Geiger, Phys. Rept. 258, 237 (1995);

S.A. Bass, B. Müller and D.K. Srivastava, manuscript in preparation.

[12] B. Zhang, C. M. Ko, B. A. Li and Z. w. Lin, Phys. Rev. C61, 067901 (2000).

[13] T. S. Biro, H. B. Nielsen, J. Knoll, Nucl. Phys. B245 (1984) 449;

J. Knoll, Z. Phys. C38 (1988) 187;

H. Sorge, M. Berenguer, H. Stöcker, W. Greiner, Phys. Lett. B289 (1992) 6;

S. E. Vance and M. Gyulassy, Phys. Rev. Lett. 83 (1999) 1735.

[14] M. Bleicher, W. Greiner, H. Stocker and N. Xu, Phys. Rev. C 62, 061901 (2000).

[15] C. Hartnack, H. Oeschler and J. Aichelin, arXiv:nucl-th/0109016.

[16] G. Q. Li and C. M. Ko, Phys. Rev. C 54, 2159 (1996);

S. Pal, C. M. Ko, Z. w. Lin and B. Zhang, Phys. Rev. C 62, 061903 (2000).

[17] J. Aichelin and C. M. Ko, Phys. Rev. Lett. 55, 2661 (1985).

[18] C. Fuchs, A. Faessler, E. Zabrodin and Y. M. Zheng, Phys. Rev. Lett. 86, 1974 (2001).

[19] C. Hartnack and J. Aichelin, SQM01 proceedings, to be published in J. Phys. G: Nucl. Part. Phys.

[20] P. Braun-Munzinger, J. Cleymans, H. Oeschler and K. Redlich, arXiv:hep-ph/0106066.

[21] H. Weber, Ph.D. thesis, Univ. of Frankfurt, 2002;

H. Weber, manuscript to be published.

[22] R. Bramm, Diplomarbeit, Johann Wolfgang Goethe-Universitt, Frankfurt am Main (2001);

T. Kollegger, Diplomarbeit, Johann Wolfgang Goethe-Universitt, Frankfurt am Main (2001).

[23] W. Cassing, E. L. Bratkovskaya and S. Juchem, Nucl. Phys. A674, 249 (2000).

[24] C. Greiner, private communication.

[25] R. Rapp and E. V. Shuryak, Phys. Rev. Lett. 86, 2980 (2001).

[26] C. Greiner and S. Leupold, J. Phys. G 27, L95 (2001).

[27] F. Wang et al., Phys. Rev. C61 (2000) 064904.

[28] J. Rafelski, B. Müller, Phys. Rev. Lett. 48, 1066 (1982); (E) 562334 (1986).

[29] P. Koch, B. Müller, J. Rafelski, Phys. Rep. 142, 167 (1986).

[30] P. Koch, B. Müller, H. Stöcker, W. Greiner, Mod. Phys. Lett. A3, 737 (1988).

[31] S. Soff, S. A. Bass, M. Bleicher, L. Bravina, E. Zabrodin, H. Stocker and W. Greiner, Phys. Lett. B471, 89 (1999).

[32] E. Andersen et al. (WA97 collaboration), Phys. Lett B433 (1998) 209.

R. Lietava et al. (WA97 collaboration), Journal of Physics G25 (1999) 181.

R. Caliandro et al. (WA97 collaboration), Journal of Physics G25 (1999) 171.

S. Margetis et al. (NA49 collaboration), Journal of Physics G25 (1999) 189.

F. Gabler et al. (NA49 collaboration), Journal of Physics G25 (1999) 199.

D. Evans et al. (WA85 and WA94 collaborations), Journal of Physics G25 (1999) 209.

[33] S. E. Vance and M. Gyulassy, Phys. Rev. Lett. 83, 1735 (1999).

[34] H. J. Drescher, J. Aichelin and K. Werner, Phys. Rev. D 65, 057501 (2002).

[35] S.A. Bass and A. Dumitru, Phys. Rev. C61 (2000) 064909.

[36] A. Dumitru, S. A. Bass, M. Bleicher, H. Stocker and W. Greiner, Phys. Lett. B460, 411 (1999). 